\title{
Postoperative Chemoradiotherapy versus Postoperative Chemotherapy for Completely Resected Gastric Cancer with D2 Lymphadenectomy: A Meta-Analysis
}

Yuan-Yuan Huang ${ }^{19}$, Qiong Yang ${ }^{29}$, Si-Wei Zhou ${ }^{2}$, Ying Wei ${ }^{2}$, Yan-Xian Chen ${ }^{2}$, De-Rong Xie ${ }^{2}$, Bei Zhang ${ }^{1 *}$

1 Department of VIP, Sun Yat-sen University Cancer Center, Sun Yat-sen University, Guangzhou, P.R. China, 2 Department of Oncology, Sun Yat-sen Memorial Hospital, Sun Yat-sen University, Guangzhou, P.R. China

\begin{abstract}
Background: Both chemoradiotherapy and chemotherapy are used in postoperative adjuvant therapy for resected gastric cancer. However, it is controversial whether chemoradiotherapy or chemotherapy is the optimal strategy for patients with gastric cancer after D2 lymphadenectomy. The present meta-analysis aims to provide more evidence on the relative benefits of adjuvant therapies in this setting.

Methods: We conducted a systematic review of randomized controlled trials, extracted time-to-event data using Tierney methods (when not reported), and performed meta-analysis to obtain the relative hazards of adjuvant chemoradiotherapy to chemotherapy on efficacy and toxicities.

Results: A total of 895 patients from 3 randomized controlled trials were identified for this meta-analysis. All patients were from Asian countries. Our results showed that postoperative chemoradiotherapy significantly improved locoregional recurrence-free survival [LRRFS: hazard ratio $(H R)=0.53,95 \% \mathrm{Cl}=0.32-0.87, p=0.01$ ] and disease-free survival (DFS: $\mathrm{HR}=0.72,95 \% \mathrm{Cl}=0.59-0.89, \mathrm{p}=0.002$ ); however, the improvement of distant metastasis recurrence-free survival (DMRFS: $\mathrm{HR}=0.86 ; 95 \% \mathrm{Cl}=0.66-1.11, \mathrm{p}=0.25)$ and overall survival (OS: $\mathrm{HR}=0.79,95 \% \mathrm{Cl}=0.61-1.03, \mathrm{p}=0.08$ ) were non-significant. The main grade 3 or 4 toxicities were equivalent between the two groups.

Conclusion: In non-selected Asian patients with resected gastric cancer who underwent D2 lymphadenectomy, postoperative chemoradiotherapy improved LRRFS and DFS but might not improve OS compared to postoperative chemotherapy.
\end{abstract}

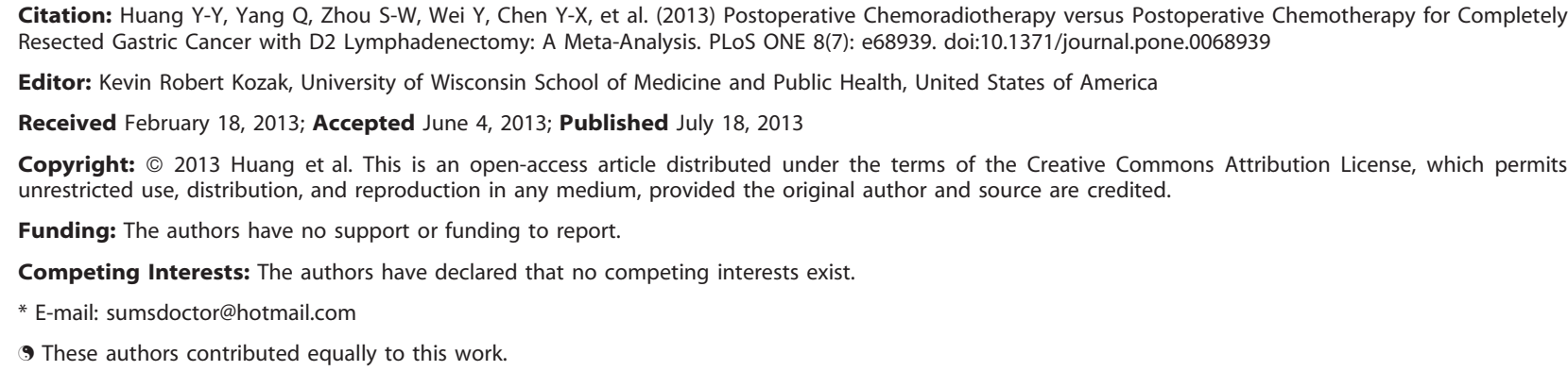

9 These authors contributed equally to this work.

\section{Introduction}

Globally, gastric cancer is the third leading cause of cancer related death among men and the fifth among women [1]. The primary curative treatment of gastric carcinoma is surgical resection [2]. Complete resection with adequate margins is widely considered as a standard goal, whereas the extent of lymph node dissection remains controversial. Irrespective of the surgical procedure used for treatment of gastric cancer, it is a consensus that patients with resected gastric cancer should receive adjuvant treatment.

In the last decade, postoperative chemoradiotherapy has become the preferred strategy for resected gastric cancer because the INT-0116 trial suggested that postoperative chemoradiotherapy had a survival advantage over observation. However, INT0116 trial has been criticized for suboptimal surgery with $54 \%$ and $36 \%$ of patients receiving D0 and D1 dissections, respectively $[3,4]$. Recently, gastrectomy with D2 lymphadenectomy has become the standard surgical procedure for curable gastric cancer in eastern Asia. It is also a recommended operation in European countries because of a reduction in gastric cancer-specific deaths with D2 dissection demonstrated in Dutch Gastric Cancer Group trial [5]. ACTS-GC and CLASSIC trials have shown that postoperative chemotherapy reduces risk of relapse and death in patients with gastric cancer after D2 lymphadenectomy [6,7]. However, still, about $10 \%$ patients eventually have local relapse after D2 curative resection [6,7]. Therefore, it is necessary to explore whether radiation added to adjuvant chemotherapy further improves survival for gastric cancer patients after D2 curative gastrectomy.

To our knowledge, there are three phase III randomized controlled trials (RCTs) which directly compared postoperative chemoradiotherapy with postoperative chemotherapy for patients with gastric cancer after D2 curative gastrectomy. Overall, two in three RCTs do not find any differences in overall survival (OS) and disease-free survival (DFS) between the two approaches $[8,9]$. 
One RCT suggests that chemoradiotherapy improves DFS compared with chemotherapy [10]. Due to the inconsistent results, we attempted to explore this issue by meta-analysis.

\section{Methods}

\section{Literature Search}

A systematic review of eligible RCTs was performed by searching the electronic databases, which consist of Cochrane Central Register of Controlled Trials, PubMed, EMBASE, ISI Web of Knowledge, Chinese biomedical literature service system (SinoMed), ASCO abstracts, and ESMO abstracts. The keywords used for search were as follow: "gastric cancer", "stomach neoplasms", "chemoradiotherapy", "chemoradiation", "chemotherapy", "D2", and "combined modality therapy". The search was limited to RCTs in English language. The deadline of this search was October 31, 2012. The reference lists of articles identified and relevant meta-analysis were searched manually to find other relevant articles. Meta-analysis was conducted according to the Preferred Reporting Items for Systematic Reviews and Meta-Analyses (PRISMA) statement [11,12]. The PRISMA checklist was showed in Checklist S1.

\section{Trial Selection and Quality Assessment}

All RCTs that compared chemoradiotherapy with chemotherapy in the setting of adjuvant therapy for resected gastric cancer with D2 lymphadenectomy were included in the present metaanalysis. If the same population appeared in other publications, the article that provided the most complete follow-up data on survival was selected. Methodological quality of the trials was assessed using a validated scale (range, 0 to 5) applied to items that influence intervention efficacy. The scale consists of items pertaining to randomization, masking, dropouts, and withdrawals, which is reported by Jadad et al [13]. A trial was regarded as high quality trial with high external and internal validities if it scored more than 3 points.

\section{Data Extraction and Analysis}

Two primary reviewers (YYH and QY) assessed all abstracts that were identified from the above-mentioned sources. Both reviewers independently selected potentially eligible abstracts according to inclusion criteria. If one of the reviewers considered an abstract potential eligible, the full text of article was retrieved and reviewed in detail by both reviewers. Disagreements were resolved by consensus or by the third reviewer (BZ). Hazard ratio (HR) and 95\% confidence interval $(95 \%$ CI) for OS, DFS, locoregional recurrence-free survival (LRRFS) and distant metastasis recurrence-free survival (DMRFS) were requested. Where published, HR and 95\% CI were extracted directly from the original article. Where HR and 95\% CI were not reported, they were calculated from published summary statistics or survival curve using Tierney method [14]. The following variables were extracted from each trial if available: total numbers of patients, age, sex, ECOG performance status, primary tumor site, Lauren classification, tumor stage, treatment regimens, endpoints, median follow-up time, Jadad scale score, and toxicities.

\section{Statistical Analysis}

The primary end points were OS, DFS, LRRFS, and DMRFS after randomization. The secondary end point was toxicity. Survival variables were defined as generic inverse variance data. We standardized the resulting treatment effect to obtain an effect size by HR. Toxicity variables were defined as dichotomous data. We standardized the outcome variable to obtain an effect size by
Risk Ratio (RR). Crude HRs and RRs with 95\% CIs were used to assess the survival benefit and risk of toxicities between chemoradiotherapy group and chemotherapy group, respectively. The significance of the pooled results was determined by the Z-test, and $\mathrm{P}<0.05$ was considered as statistically significant.

Heterogeneity assumption was checked by a chi-square-based Q-test and also expressed as $\mathrm{I}^{2}$. A P-value of more than 0.10 for the Q-test and $\mathrm{I}^{2}$ of less than $50 \%$ indicated a lack of heterogeneity across the trials. If $\mathrm{P}$-value of heterogeneity test was more than 0.1 and $\mathrm{I}^{2}$ was less than $50 \%$, fixed effect model was performed and random effect model was used vice versa. However, due to the fixed effect model tended to underestimate standard errors of pooled estimates, random effect model was used for the quantitative pooling [15]. An estimate of the potential publication bias was carried out by funnel plot. An asymmetric plot suggested a possible publication bias. The funnel plot asymmetry was assessed by Egger's test. $\mathrm{P}<0.05$ was considered representative of statistically significant publication bias [16]. The statistical tests for our meta-analysis were performed with RevMan software (version 5.1, Cochrane) and STATA version 10.0 (Stata Corporation, College Station, TX).

\section{Results}

\section{Trial Flow, Characteristics, and Quality Appraisal}

Figure 1 was the flow chart of RCTs selection for meta-analysis. A total of 895 patients from 3 RCTs were identified for this metaanalysis at last [8-10]. All patients were from Asian countries. Only one RCT uses intensive modulation radiotherapy (IMRT) as a part of concurrent chemoradiotherapy and shows DFS benefit from chemoradiotherapy [10]. One RCT uses capecitabine combined with cisplatin as chemotherapy regimen [8], the other two RCTs use the same chemotherapy regimen as that of INT$0116[9,10]$. All RCTs don't show that chemoradiotherapy has an OS advantage over chemotherapy. Table 1 and Table 2 showed important baseline characteristics and Jadad scores of selected trials.

\section{Efficacy: LRRFS, DMRFS, DFS, and OS}

895 randomized patients from 3 RCTs, 457 in the chemoradiotherapy group and 438 in the chemotherapy group, were included in the meta-analyses of LRRFS, DMRFS, and DFS. 437 randomized patients from 2 RCTs, 227 in the chemoradiotherapy group and 210 in the chemotherapy group were included in the meta-analysis of OS. The result of the test for heterogeneity of the treatment effects were not significant $(\mathrm{P}>0.10)$. Compared to chemotherapy, chemoradiotherapy significantly reduced the risk of locoregional recurrence and disease recurrence by $47 \%$ $(\mathrm{P}=0.01)$ and $28 \%(\mathrm{P}=0.001)$, respectively. However, chemoradiotherapy didn't significantly improve DMRFS $(\mathrm{P}=0.26)$ and OS $(\mathrm{P}=0.07)$. The detailed data was shown in Figure 2.

\section{Toxicities}

Overall, toxicities in 3 selected RCTs were tolerable. The most common grade 3 or 4 adverse events were nausea, vomiting, hand and foot syndrome (only occurred in patients received capecitabine) and neutropenia. Pooled results suggested that there was no significant difference between two treatment approaches (Figure 3).

\section{Publication Bias Assessment}

Begg's funnel plot and Egger's test were performed to access the publication bias of literatures. The shapes of the funnel plots did not reveal any evidence of obvious asymmetry (Figure 4). Then, the Egger's test was used to provide statistical evidence of funnel 


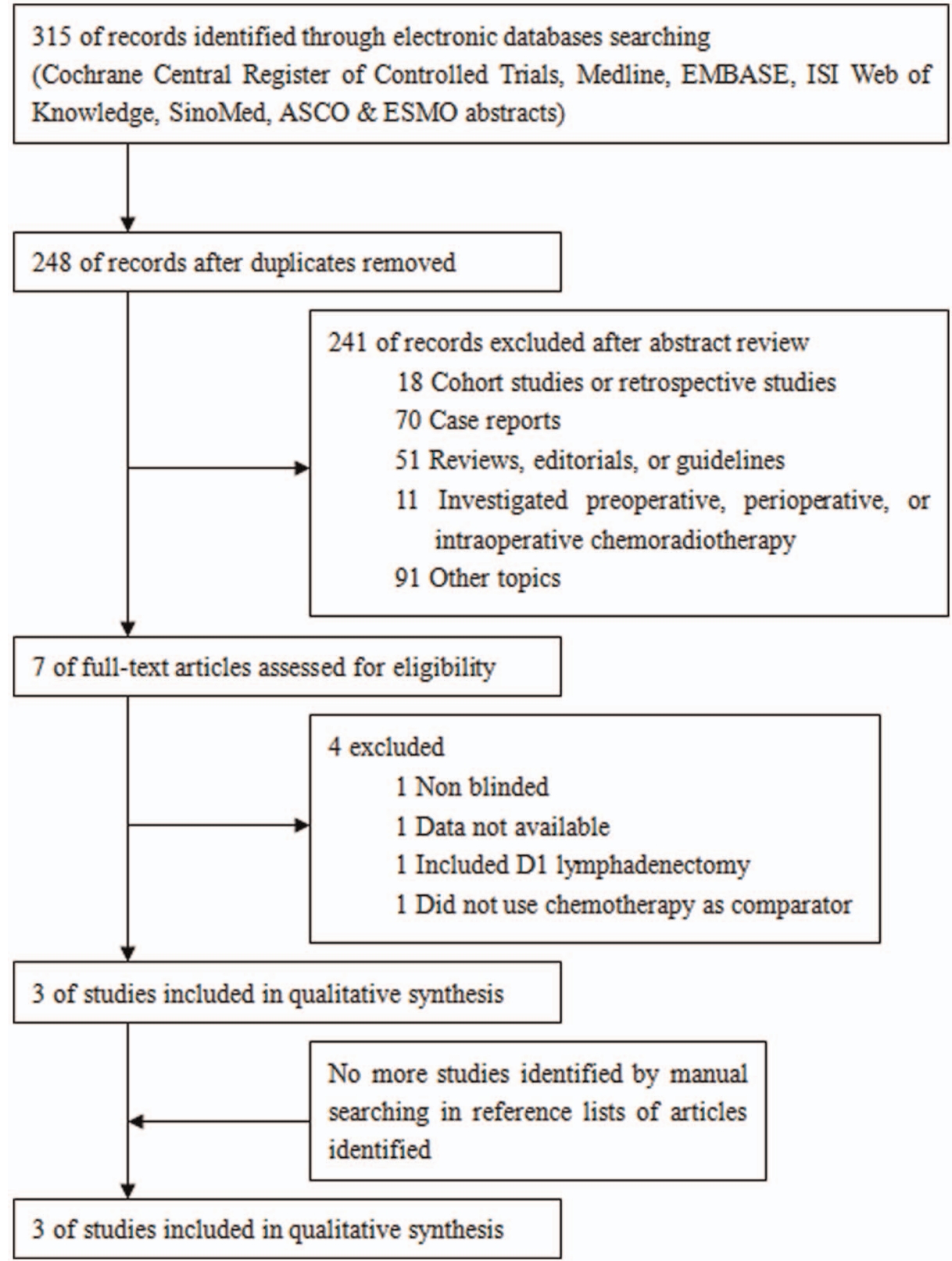

Figure 1. Flow chart of randomized controlled trials selection. doi:10.1371/journal.pone.0068939.g001

plot symmetry. The results still did not suggest any evidence of publication bias $(\mathrm{Z}=1.04, \mathrm{P}=0.30$ for LRRFS, DMRFS, and DFS, $Z=0, P=1.00$ for $\mathrm{OS}$, respectively).

\section{Sensitivity Analyses}

Sensitivity analyses were performed to evaluate whether the pooled estimates of LRRFS, DMRFS, DFS, and OS were different by exclusion of the highest weighted study and by omitting the trial that only included III/IV gastric cancer in each pooled analysis. Finally, the results were all consistent with the above outcomes.

\section{Discussion}

Now, more and more surgeons accept D2 gastrectomy as required operation for patients with resectable gastric cancer. The optimal adjuvant therapy strategy is not well-defined in these patients. A Korean observational study suggests that postoperative chemoradiotherapy can prolong survival and decrease recurrence compared to observation [17]. In contrast, both single RGT and IPD-based meta-analysis suggest a survival benefit associated with postoperative chemotherapy $[6,7,18]$. Therefore, it is valuable to explore relative benefits of adjuvant therapies in this setting. Overall, our meta-analysis showed that postoperative chemoradiotherapy improved LRRFS and DFS, but didn't improve DMRFS and OS compared with postoperative chemotherapy. To our knowledge, this was the first meta-analysis that compared 
Table 1. Characteristics of the selected RCTs.

\begin{tabular}{|c|c|c|c|c|c|c|}
\hline \multirow{2}{*}{ References Characteristics } & \multicolumn{2}{|l|}{ Lee et al 2012} & \multicolumn{2}{|c|}{ Kim et al 2012} & \multicolumn{2}{|c|}{ Zhu et al 2012} \\
\hline & CT & CRT & CT & CRT & CT & CRT \\
\hline Total number & 228 & 230 & 44 & 46 & 165 & 186 \\
\hline Age year(range) & $56(22-77)$ & $56(28-76)$ & NR & NR & $59(42-75)$ & $56(38-73)$ \\
\hline \multicolumn{7}{|l|}{ Sex No. (\%) } \\
\hline Male & 153(67.1) & $143(62.2)$ & $25(56.8)$ & $34(73.9)$ & $126(76.4)$ & $135(72.9)$ \\
\hline Female & $75(32.9)$ & $87(37.8)$ & 19(43.2) & $12(26.1)$ & $39(23.6)$ & $51(27.1)$ \\
\hline \multicolumn{7}{|l|}{ ECOG PS No. (\%) } \\
\hline 0 & $96(42.1)$ & $99(43.0)$ & $27(61.4)$ & $35(76.1)$ & NR & NR \\
\hline 1 & 132(57.9) & $131(57.0)$ & $17(38.6)$ & $11(23.9)$ & NR & NR \\
\hline \multicolumn{7}{|l|}{ Primary tumor site No.(\%) } \\
\hline Proximal & 9(3.9) & 13(5.7) & $2(4.5)$ & $3(6.5)$ & $15(9.1)$ & $30(16.1)$ \\
\hline Body & $112(49.1)$ & $107(46.5)$ & 19(43.2) & $26(56.5)$ & $33(20)$ & $21(11.3)$ \\
\hline Antrum & $87(38.2)$ & $90(39.1)$ & $18(40.9)$ & $14(30.5)$ & $117(70.9)$ & $135(72.6)$ \\
\hline Multiple/diffuse & $20(8.8)$ & $20(8.7)$ & $5(11.4)$ & $3(6.5)$ & 0 & 0 \\
\hline \multicolumn{7}{|l|}{ Lauren classification No. (\%) } \\
\hline Intestinal & $88(38.6)$ & $75(32.6)$ & 15(34.1) & $16(34.8)$ & NR & NR \\
\hline Diffuse & $130(57.0)$ & 144(62.6) & $24(54.5)$ & $26(56.5)$ & NR & NR \\
\hline Mixed & $6(2.6)$ & $8(3.5)$ & $3(6.8)$ & $2(4.3)$ & NR & NR \\
\hline Not specified & $4(1.8)$ & $3(1.3)$ & $2(4.5)$ & $2(4.3)$ & NR & NR \\
\hline \multicolumn{7}{|l|}{ Tumor stage No. (\%) } \\
\hline IB & $50(21.9)$ & $49(21.3)$ & 0 & 0 & 15(9.1) & $20(10.8)$ \\
\hline II & $86(37.7)$ & $84(36.5)$ & 0 & 0 & $30(18.2)$ & $36(19.4)$ \\
\hline III & 65(28.6) & $71(30.8)$ & $31(75)$ & $34(73.9)$ & $96(58.2)$ & 103(55.4) \\
\hline IV(M0) & $27(11.8)$ & $26(11.3)$ & $11(25)$ & $12(26.1)$ & $24(14.5)$ & $27(14.5)$ \\
\hline Treatment regimens & XP\# & $\mathrm{XP} / \mathrm{XRT} / \mathrm{XP} *$ & $F L ※$ & $\mathrm{FL} / \mathrm{RT}^{\S}$ & $\mathrm{FL} ※$ & FL/IMRT" \\
\hline \multirow[t]{2}{*}{ Endpoints } & \multicolumn{2}{|c|}{ 3-ys DFS: $74.2 \%$ vs $78.2 \%, p=0.0862$} & \multicolumn{2}{|c|}{5 -ys DFS: $50.0 \%$ vs $60.9 \%, p=0.246$} & \multicolumn{2}{|c|}{5 -ys RFS: $35.8 \%$ vs $45.2 \%, p=0.029$} \\
\hline & \multicolumn{2}{|c|}{ OS not reached when data analyzed } & \multicolumn{2}{|c|}{5 -ys OS: $54.6 \%$ vs $65.2 \%, p=0.67$} & \multicolumn{2}{|c|}{5 -ys OS: $41.8 \%$ vs $48.4 \%, p=0.122$} \\
\hline Follow-up month Median(range) & \multicolumn{2}{|l|}{$53.2(36.9-77.3)$} & \multicolumn{2}{|c|}{$86.7(60.3-116.5)$} & \multicolumn{2}{|l|}{42.5} \\
\hline Jadad scale score & \multicolumn{2}{|l|}{3} & \multicolumn{2}{|l|}{3} & \multicolumn{2}{|l|}{3} \\
\hline
\end{tabular}

CT: chemotherapy, CRT: chemoradiotherapy, NR: not reported, 3-ys DFS: 3-year disease-free survival, 5-ys DFS: 5-year disease-free survival, 5-ys RFS: 5-year recurrencefree survival, 5-ys OS: 5-year overall survival.

\#XP regimen: capecitabine $1000 \mathrm{mg} / \mathrm{m} 2$ twice daily on days 1 to 14; cisplatin $60 \mathrm{mg} / \mathrm{m} 2$ on day 1 every 3 weeks, totally 6 cycles.

*XP/XRT/XP: Two cycles of XP, then XRT (45 Gy of radiation at 1.8 Gy per day, 5 days per week, for 5 weeks with continuous capecitabine 825 mg/m2 twice daily during radiotherapy), followed by two cycles of XP.

*FL regimen: 5 -fu $425 \mathrm{mg} / \mathrm{m} 2$, leucovorin $20 \mathrm{mg} / \mathrm{m} 2$, for 5 days with a 4-week interval, totally 5 cycles.

${ }^{\S} \mathrm{FL} / \mathrm{RT}$ : 1 cycle of FL, then RT(45 Gy of radiation at 1.8 Gy per day, 5 days per week, for 5 weeks with 2 cycles of FL), followed by two cycles of FL.

"FL/IMRT: 1 cycle of FL, then IMRT ( 45 Gy of radiation at 1.8 Gy per day, 5 days per week, for 5 weeks with 2 cycles of FL), followed by two cycles of FL.

doi:10.1371/journal.pone.0068939.t001

Table 2. Main grade $3 / 4$ toxicities of selected RCTs.

\begin{tabular}{|c|c|c|c|c|c|c|}
\hline References & Regimens & $\mathbf{N}$ & Nausea/Vomit n (\%) & Neutropenia n (\%) & Anemia n (\%) & Thrombocyto- penia n (\%) \\
\hline \multirow[t]{2}{*}{ Lee et al } & XP/XRT & 230 & $35(15.4)$ & $110(48.4)$ & $1(0.4)$ & $2(0.9)$ \\
\hline & $X P$ & 228 & $36(15.9)$ & $92(40.7)$ & $4(1.7)$ & $0(0)$ \\
\hline \multirow[t]{2}{*}{ Kim et al } & $\mathrm{FL} / \mathrm{RT}$ & 41 & * & * & * & * \\
\hline & $\mathrm{FL}$ & 45 & * & $*$ & * & * \\
\hline \multirow[t]{2}{*}{ Zhu et al } & $\mathrm{FL} / \mathrm{IMRT}$ & 186 & $5(4.3)$ & $14(7.5)$ & $0(0)$ & $0(0)$ \\
\hline & $\mathrm{FL}$ & 165 & $0(0)$ & $12(7.3)$ & $0(0)$ & $0(0)$ \\
\hline
\end{tabular}

XP: capecitabine+cisplatin, XRT: radiotherapy with capecitabine, FL: fluorouracil plus leucovorin, RT: radiotherapy, IMRT: intensive modulation radiotherapy;

*Grade $3 / 4$ hematologic and gastrointestinal toxicities occurred in $19.6 \%$ and $17.4 \%$ in the chemoradiotherapy arm and $25 \%$ and $11.4 \%$ in the chemotherapy arm, respectively.

doi:10.1371/journal.pone.0068939.t002 


\begin{tabular}{|c|c|c|c|c|c|c|c|c|c|c|}
\hline Studv or Subgroup & log[Hazard Ratio] & SE & $\begin{array}{l}\text { CRT } \\
\text { Total } \\
\end{array}$ & $\begin{array}{l}\text { CT } \\
\text { Total }\end{array}$ & Weight & $\begin{array}{l}\text { Hazard Ratio } \\
\text { IV. Random, } 95 \% \mathrm{Cl}\end{array}$ & & $\begin{array}{r}\text { Haza } \\
\text { IV, Ranc }\end{array}$ & $\begin{array}{l}\text { ard Ratio } \\
\text { dom. } 95 \% \mathrm{Cl}\end{array}$ & \\
\hline \multicolumn{11}{|l|}{ 1.1.1 LRRFS } \\
\hline Kim TH 2012 & -1.409 & 0.5123 & 41 & 45 & $19.8 \%$ & $0.24[0.09,0.67]$ & $\longleftrightarrow$ & & & \\
\hline Lee J 2012 & -0.3649 & 0.3924 & 230 & 228 & $29.4 \%$ & $0.69[0.32,1.50]$ & & & & \\
\hline Zhu WG 2012 & -0.4951 & 0.2439 & 186 & 165 & $50.7 \%$ & $0.61[0.38,0.98]$ & & & & \\
\hline Subtotal $(95 \% \mathrm{Cl})$ & & & 457 & 438 & $100.0 \%$ & $0.53[0.32,0.87]$ & & & & \\
\hline \multicolumn{11}{|c|}{$\begin{array}{l}\text { Heterogeneity: } \mathrm{Tau}^{2}=0.07 ; \mathrm{Chi}^{2}=3.04, \mathrm{df}=2(\mathrm{P}=0.22) ; \mathrm{I}^{2}=34 \% \\
\text { Test for overall effect: } \mathrm{Z}=2.48(\mathrm{P}=0.01)\end{array}$} \\
\hline \multicolumn{11}{|l|}{ 1.1.2 DMRFS } \\
\hline Kim TH 2012 & -0.3423 & 0.3229 & 41 & 45 & $16.7 \%$ & $0.71[0.38,1.34]$ & & & & \\
\hline Lee J 2012 & -0.1167 & 0.1978 & 230 & 228 & $44.5 \%$ & $0.89[0.60,1.31]$ & & & & \\
\hline Zhu WG 2012 & -0.1124 & 0.212 & 186 & 165 & $38.8 \%$ & $0.89[0.59,1.35]$ & & & & \\
\hline Subtotal $(95 \% \mathrm{Cl})$ & & & 457 & 438 & $100.0 \%$ & $0.86[0.66,1.11]$ & & & & \\
\hline \multicolumn{11}{|c|}{$\begin{array}{l}\text { Heterogeneity: } \mathrm{Tau}^{2}=0.00 ; \mathrm{Chi}^{2}=0.41, \mathrm{df}=2(\mathrm{P}=0.81) ; \mathrm{I}^{2}=0 \% \\
\text { Test for overall effect: } Z=1.16(P=0.25)\end{array}$} \\
\hline \multicolumn{11}{|l|}{ 1.1.3 DFS } \\
\hline Kim TH 2012 & -0.4833 & 0.3118 & 41 & 45 & $11.1 \%$ & $0.62[0.33,1.14]$ & & & & \\
\hline Lee J 2012 & -0.308 & 0.1791 & 230 & 228 & $33.6 \%$ & $0.73[0.52,1.04]$ & & & & \\
\hline Zhu WG 2012 & -0.3001 & 0.1396 & 186 & 165 & $55.3 \%$ & $0.74[0.56,0.97]$ & & & & \\
\hline Subtotal $(95 \% \mathrm{Cl})$ & & & 457 & 438 & $100.0 \%$ & $0.72[0.59,0.89]$ & & & & \\
\hline \multicolumn{11}{|c|}{$\begin{array}{l}\text { Heterogeneity: } \operatorname{Tau}^{2}=0.00 ; \mathrm{Chi}^{2}=0.30, \mathrm{df}=2(P=0.86) ; \mathrm{I}^{2}=0 \% \\
\text { Test for overall effect: } Z=3.11(P=0.002)\end{array}$} \\
\hline \multicolumn{11}{|l|}{ 1.1.4 os } \\
\hline Kim TH 2012 & -0.2714 & 0.3393 & 41 & 45 & $15.4 \%$ & $0.76[0.39,1.48]$ & & & & \\
\hline Zhu WG 2012 & -0.2241 & 0.1446 & 186 & 165 & $84.6 \%$ & $0.80[0.60,1.06]$ & & & & \\
\hline Subtotal $(95 \% \mathrm{Cl})$ & & & 227 & 210 & $100.0 \%$ & $0.79[0.61,1.03]$ & & & & \\
\hline \multicolumn{11}{|c|}{$\begin{array}{l}\text { Heterogeneity: } \mathrm{Tau}^{2}=0.00 ; \mathrm{Chi}^{2}=0.02, \mathrm{df}=1(P=0.90) ; \mathrm{I}^{2}=0 \% \\
\text { Test for overall effect: } Z=1.74(P=0.08)\end{array}$} \\
\hline
\end{tabular}

Figure 2. Forest plot of efficacy comparing chemoradiotherapy (CRT) with chemotherapy (CT). doi:10.1371/journal.pone.0068939.g002

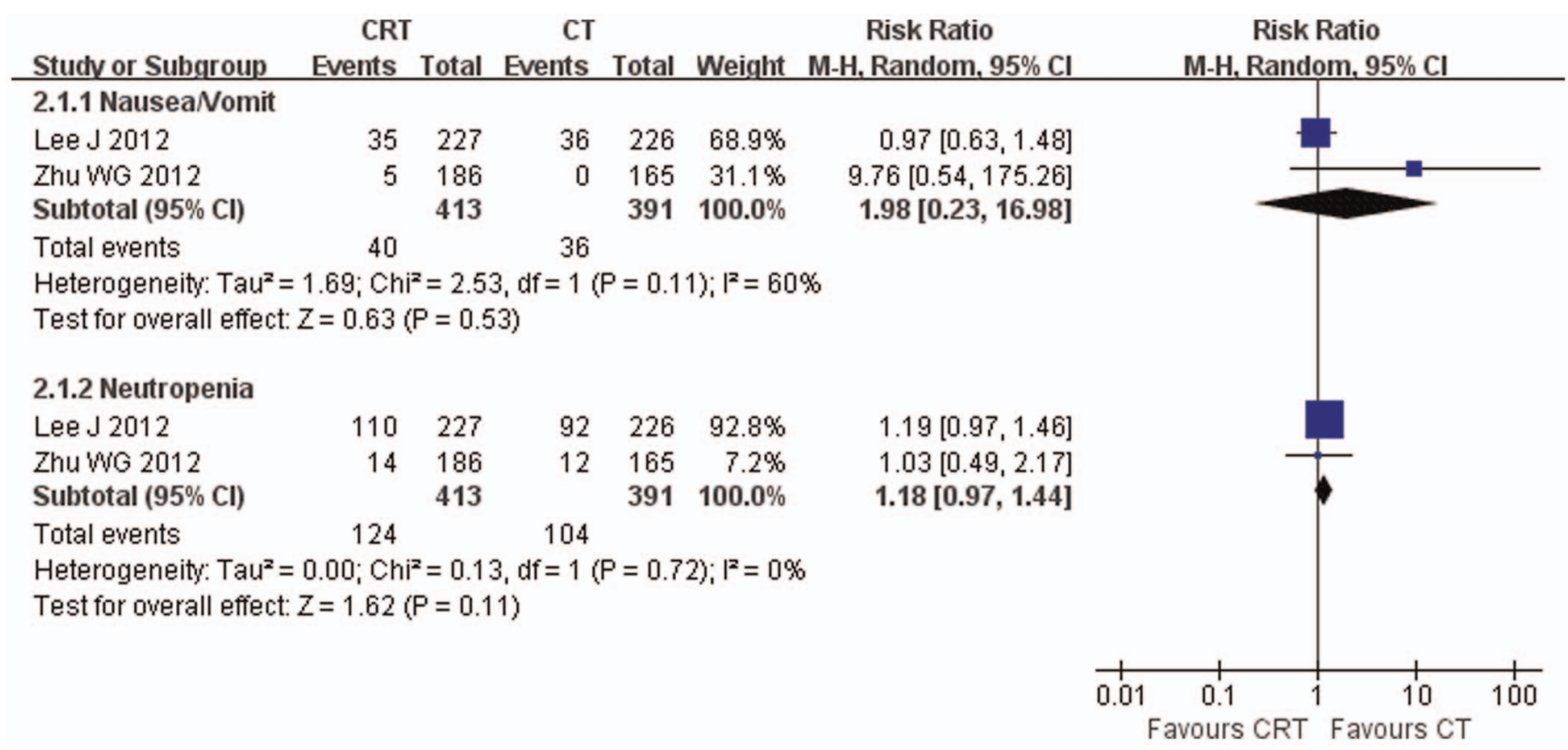

Figure 3. Forest plot of toxicities comparing chemoradiotherapy (CRT) with chemotherapy (CT). doi:10.1371/journal.pone.0068939.g003 


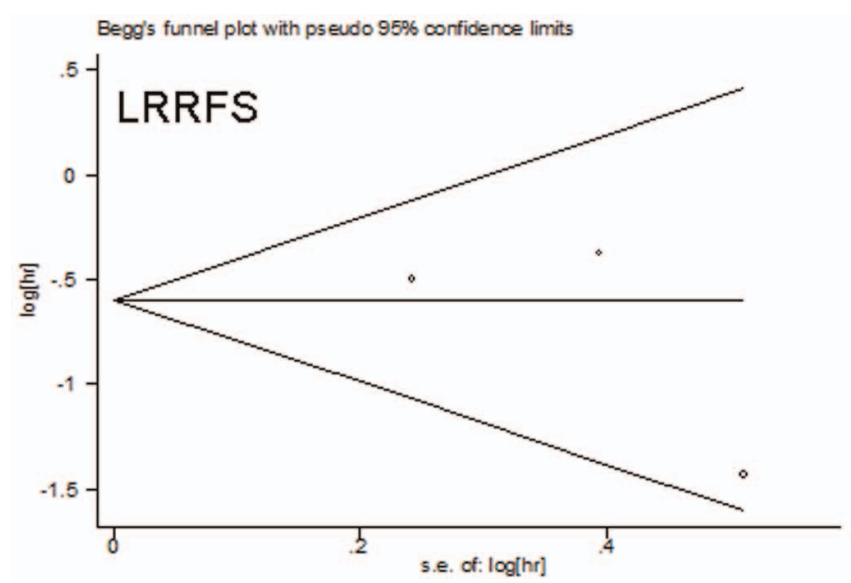

Beg's funnel plot with ps eudo $95 \%$ confidence limits

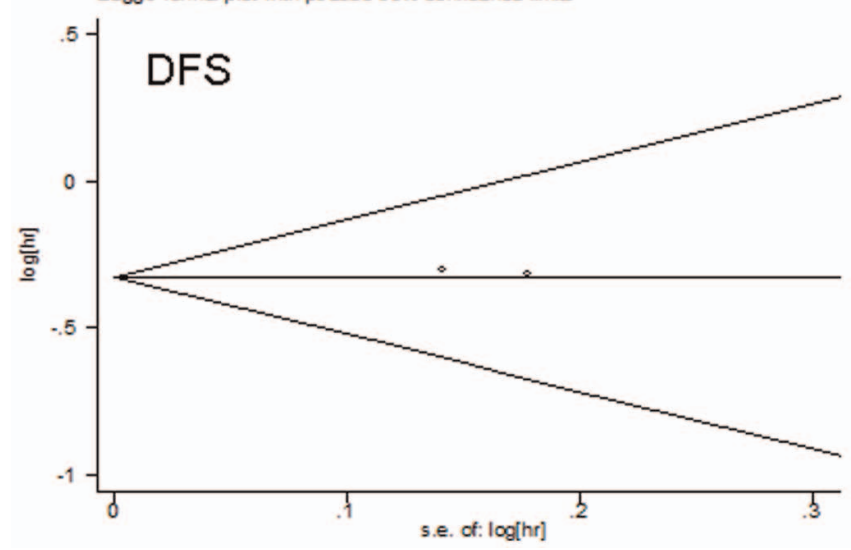

Figure 4. Begg's funnel plot for publication bias. doi:10.1371/journal.pone.0068939.g004

postoperative chemoradiotherapy with postoperative chemotherapy in resected gastric cancer with D2 lymphadenectomy.

Although chemoradiotherapy improved LRRFS and DFS, good locoregional control didn't transfer to OS benefit. Can we conclude that no survival benefit of chemoradiotherapy is principally a consequence of no DMRFS benefit? To answer this question, three facts should be emphasized. First, compared with D1 lymphadenectomy, D2 or more extended lymphadenectomy produces more reduction of local recurrence than that of distant metastasis. For example, in Dutch Gastric Cancer Group Trial, the locoregional recurrence is reduced by $13 \%(58 \%$ D1 group vs. $45 \%$ D2 group), which is higher than $7 \%$ of reduction in distant metastasis (48\% D1 group vs. 41\% D2 group). In a Taiwanese trial, the locoregional recurrence is reduced by $11 \%(30 \% \mathrm{D} 1$ group vs. 19\% D3 group), which is higher than $8 \%$ of reduction in distant metastasis (37\% Dl group vs. 29\% D3 group) $[5,19]$. Second, predominant recurrence pattern associated with D2 lymphadenectomy was distant metastasis in Asian population [20]. In contrast, locoregional recurrence was more frequent than distant metastasis in West countries population who underwent D2 gastrectomy [21]. This viewpoint was also supported by results of RCTs selected in our present meta-analysis, in which distant metastasis rate is higher than local recurrence rate for Asian population $(22.5 \%-43.3 \%$ vs. $6.6 \%-23 \%)$. At last, whatever type of lymphadenectomy is performed, postoperative chemoradiotherapy doesn't reduce distant metastasis even compared with observation $[3,4,17]$. Taken together, bad DMRFS might offset
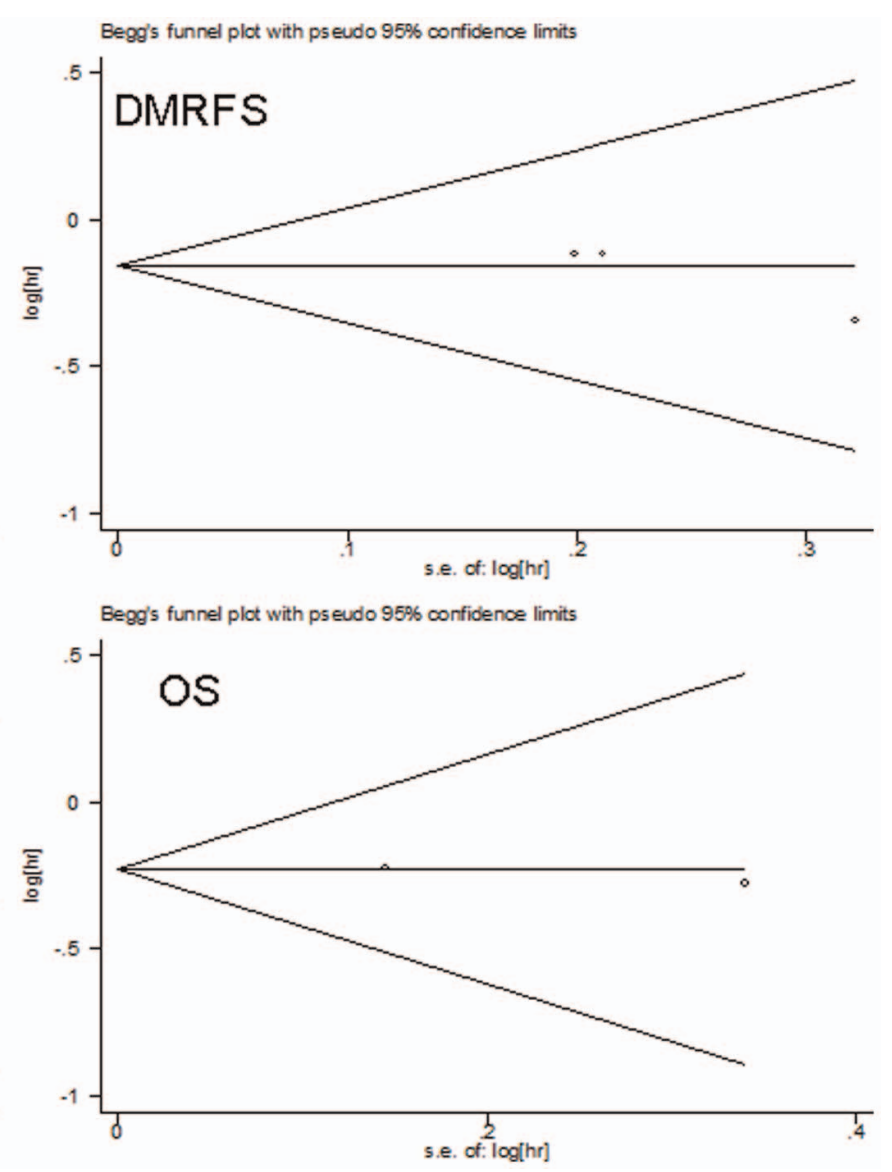

LRRFS benefit from chemoradiotherapy for Asian patients underwent D2 gastrectomy.

To date, the reason that distant metastasis rate is higher than local recurrence rate for Asian population with gastric cancer after D2 lymphadenectomy is not very clear. A meta-analysis showed that there was a high percentage of diffuse-type histology gastric cancer in Asian population, which accounted for 50\% at least [22]. Diffuse gastric cancer is prone to early metastasis, and for whom chemoradiotherapy does not appear to confer a benefit [23]. In present meta-analysis, patients were from Asian countries and diffuse-type gastric cancer accounted for more than $50 \%$ in two trials $[8,9]$. We can't exclude that more diffuse-type gastric cancer selected in the two original trials was the main reason for higher rate of distant metastasis than that of locoregional recurrence. As commented by Brooks, if the finding of decreased efficacy of chemoradiotherapy in diffuse histology is confirmed, future trials may consider exploring different adjuvant approaches based on histology [23].

Our meta-analysis didn't show that reduction of locoregional recurrence could transfer to OS benefit by adding radiation to postoperative chemotherapy in non-selected population. How is the result if we focused on subgroup of patients with pathologic lymph node metastasis at the time of surgery? A retrospective study shows that adjuvant chemoradiotherapy is associated with a significant improvement in survival for subgroup of patients with node-positive gastric cancer treated with D2 lymphadenectomy [24]. Subgroup analysis of ARTIST trial also shows that patients randomly assigned to the chemoradiotherapy arm experienced 
superior DFS when compared with those who received chemotherapy alone [8]. In contrast, in another Korean RCT which almost included patients purely with pathologic lymph node metastasis, intent-to-treat analysis doesn't show that addition of radiation therapy to chemotherapy significantly improves DFS or OS [9]. Due to the inconsistent result, we couldn't get a definite conclusion on the benefit of adjuvant chemoradiotherapy for patients with pathologic lymph node metastasis. We hoped that the ongoing ARTIST-II trial will give us a clear answer.

The main grade 3 or 4 toxicities were nausea/vomit and neutropenia irrespective of chemoradiotherapy or chemotherapy. Overall, meta-analysis didn't find any difference in toxicities between two treatment approaches.

Although this meta-analysis was based on high-quality RCTs and was properly conducted, there are some typical limitations in our study. One major limitation is the number of trials is quite small and that possibly could not unveil the real situation, but the sample size of patients is amounted to 895. Another, all of the data was extracted from abstracted data (AD) instead of individual patient data (IPD), which would be less powerful to confirm our findings. However, a correlation analysis shows AD meta-analysis is strongly correlated with IPD meta-analysis [25], indicating AD as a kind of acceptable and practical method of meta-analysis alternative for IPD. The third, characteristics of patients were similar among selected trials, except for tumor stage. Stage I B-IV included in two selected trials $[8,10]$, in contrast with only stage III/IV gastric cancer was included in Kim et al's trial [9]. However, the result of meta-analysis was not materially altered after omitting this trial (sensitivity analyses). In addition, the possible existence of unpublished studies should be aware of, which could lead to potential

\section{References}

1. Jemal A, Bray F, Center MM, Ferlay J, Ward E, et al. (2011) Global cancer statistics. CA Cancer J Clin 61: 69-90.

2. Macdonald JS (2005) Role of post-operative chemoradiation in resected gastric cancer. J Surg Oncol 90: 166-170.

3. Macdonald JS, Smalley SR, Benedetti J, Hundahl SA, Estes NG, et al. (2001) Chemoradiotherapy after surgery compared with surgery alone for adenocarcinoma of the stomach or gastroesophageal junction. N Engl J Med 345: 725-730.

4. Smalley SR, Benedetti JK, Haller DG, Hundahl SA, Estes NC, et al. (2012) Updated analysis of SWOG-directed intergroup study 0116: a phase III trial of adjuvant radiochemotherapy versus observation after curative gastric cancer resection. J Clin Oncol 30: 2327-2333.

5. Hartgrink HH, van de Velde CJ, Putter H, BonenkampJJ, Klein Kranenbarg E, et al. (2004) Extended lymph node dissection for gastric cancer: who may benefit? Final results of the randomized Dutch gastric cancer group trial. J Clin Oncol 22: 2069-2077.

6. Sasako M, Sakuramoto S, Katai H, Kinoshita T, Furukawa H, et al. (2011) Fiveyear outcomes of a randomized phase III trial comparing adjuvant chemotherapy with S-1 versus surgery alone in stage II or III gastric cancer. J Clin Oncol 29: 4387-4493.

7. Bang YJ, Kim YW, Yang HK, Chung HC, Park YK, et al. (2012) Adjuvant capecitabine and oxaliplatin for gastric cancer after D2 gastrectomy (CLASSIC): a phase 3 open-label, randomised controlled trial. Lancet 379: 315-321.

8. Lee J, Lim do H, Kim S, Park SH, Park JO, et al. (2012) Phase III trial comparing capecitabine plus cisplatin versus capecitabine plus cisplatin with concurrent capecitabine radiotherapy in completely resected gastric cancer with D2 lymph node dissection: the ARTIST trial. J Clin Oncol 30: 268-273.

9. Kim TH, Park SR, Ryu KW, Kim YW, Bae JM, et al. (2012) Phase 3 Trial of Postoperative Chemotherapy Alone Versus Chemoradiation Therapy in Stage III-IV Gastric Cancer Treated With R0 Gastrectomy and D2 Lymph Node Dissection. Int J Radiat Oncol Biol Phys 84: e585-592.

10. Zhu WG, Xua DF, Pu J, Zong CD, Li T, et al. (2012) A randomized, controlled, multicenter study comparing intensity-modulated radiotherapy plus concurrent chemotherapy with chemotherapy alone in gastric cancer patients with D2 resection. Radiother Oncol 104: 361-366.

11. Moher D, Liberati A, Tetzlaff J, Altman DG, PRISMA Group (2009) Preferred reporting items for systematic reviews and meta-analyses: the PRISMA statement. PLoS Med 6: e1000097.

12. Qi WX, Shen Z, Lin F, Sun YJ, Min DL, et al. (2013) Overall survival benefits for irinotecan-containing regimens as first-line treatment for advanced gastric cancer: an updated meta-analysis of ten randomized controlled trials. Int J Cancer 132: E66-73. publication bias. However, no such bias was found by statistical methods. In general, regarding these limitations mentioned above, we should interpret the results with adequate caution.

In a summary, postoperative chemoradiotherapy might have no survival advantage over postoperative chemotherapy for nonselected Asian population with curable gastric cancer after D2 lymphadenectomy. However, diffuse-type histology and positive lymph node disease might have an important impact on patients benefit from different adjuvant therapies. At the present, limited number of trials limited further subgroup analysis to confirm our speculation. Future trials may consider exploring different adjuvant approaches for patients after D2 gastrectomy based on histology and lymph node status.

\section{Supporting Information}

\section{Checklist S1}

(DOC)

\section{Acknowledgments}

We thank the authors who kindly provided the data necessary for our study. We also thank Dr. Hui-Lin Chen, The Hong Kong Polytechnic University for her critical reading and editing to the manuscript.

\section{Author Contributions}

Conceived and designed the experiments: CV MB. Performed the experiments: GD EO BP CV MM. Analyzed the data: CV MB EO BP LE. Wrote the paper: CV MB.

13. Jadad AR, Moore RA, Carroll D, Jenkinson C, Reynolds DJ, et al. (1996) Assessing the quality of report of randomized clinical trials: Is blinding necessary? Controlled Clin Trials 17: 1-12.

14. Tierney JF, Stewart LA, Ghersi D, Burdett S, Sydes MR (2007) Practical methods for incorporating summary time-to-event data into meta-analysis. Trials 8: 16 .

15. Lau J, Ioannidis JP, Schmid CH (1997) Quantitative synthesis in systematic reviews. Ann Intern Med 127: 820-826.

16. Egger M, Davey Smith G, Schneider M, Minder C (1997) Bias in meta-analysis detected by a simple, graphical test. BMJ 315: 629-634.

17. Kim S, Lim DH, Lee J, Kang WK, MacDonald JS, et al. (2005) An observational study suggesting clinical benefit for adjuvant postoperative chemoradiation in a population of over 500 cases after gastric resection with D2 nodal dissection for adenocarcinoma of the stomach. Int J Radiat Oncol Biol Phys 63: 1279-1285.

18. GASTRIC (Global Advanced/Adjuvant Stomach Tumor Research International Collaboration) Group, Paoletti X, Oba K, Burzykowski T, Michiels S, et al. (2010) Benefit of adjuvant chemotherapy for resectable gastric cancer: a meta-analysis. JAMA 303: 1729-1737.

19. Wu CW, Hsiung CA, Lo SS, Hsieh MC, Chen JH, et al. (2006) Nodal dissection for patients with gastric cancer: a randomized controlled trial. Lancet Oncol 7: 309-315.

20. Tsujinaka T, Fujitani K, Hirao M, Kurokawa Y (2008) Current status of chemoradiotherapy for gastric cancer in Japan. Int J Clin Oncol 13: 117-120.

21. Cirera L, Balil A, Batiste-Alentorn E, Tusquets I, Cardona T, et al. (1999) Randomized clinical trial of adjuvant mitomycin plus tegafur in patients with resected stage III gastric cancer. J Clin Oncol 17: 3810-3815.

22. Hsu C, Shen YC, Cheng CC, Cheng AL, Hu FC, et al. (2012) Geographic difference in safety and efficacy of systemic chemotherapy for advanced gastric or gastroesophageal carcinoma: a meta-analysis and meta-regression. Gastric Cancer 15: 265-280.

23. Brooks GA, Enzinger PC, Fuchs CS (2012) Adjuvant therapy for gastric cancer: revisiting the past to clarify the future. J Clin Oncol 30: 2297-2299.

24. Costa WL Jr, Coimbra FJ, Fogaroli RC, Ribeiro HS, Diniz AL, et al. (2012) Adjuvant chemoradiotherapy after d2-lymphadenectomy for gastric cancer: the role of n-ratio in patient selection. results of a single cancer center. Radiat Oncol 7: 169.

25. Bria E, Gralla RJ, Raftopoulos H, Giannarelli D (2007) Comparing two methods of meta-analysis in clinical research - individual patient data-based (IPD) and literature-based abstracted data (AD) methods: Analyzing five oncology issues involving more than 10,000 patients in randomized clinical trials (RCTs). J Clin Oncol. 2007 ASCO Annual Meeting Proceedings Part I; 25: 6512. 